\title{
BILATERAL EXUDATIVE RETINAL DETACHMENT DUE TO HYPERTENSIVE RETINOPATHY AND CHOROIDOPATHY IN YOUNG PATIENT WITH CHRONIC KIDNEY DISEASE
}

\author{
Indha Dwi Kartikasari, Nadia Artha Dewi, Mirza Metita, Safaruddin Refa \\ Department of Ophthalmology, Universitas Brawijaya, Saiful Anwar Hospital Malang
}

\begin{abstract}
Introduction: Severe systemic hypertension in chronic kidney disease can cause significant damage to the eye. Although hypertensive retinopathy is a well-known complication, hypertensive optic neuropathy and choroidopathy are much less common. The aim of this study is to report retinal manifestation in young patient with chronic kidney disease.
\end{abstract}

Method: A 26-year-old man with underlying disease chronic kidney disease (CKD) gr-V underwent bilateral bullous exudative retinal detachments. Retinal arteriolar narrowing, vascular tortuosity, arteriovenous nicking, optic disc swelling, retinal haemorrhage, elschnig spot, siegrist streak were identified in both eyes. Blood pressure was $200 / 140 \mathrm{mmHg}$ with visual acuity 0,5/60 OU. The patient was diagnosed with bilateral hypertensive retinopathy and choroidopathy with bulous exudative retinal detachments.

Results: After antihypertensive treatment, visual acuity improved, but the exudative retinal detachments and retinal hemorrhages reduced. A patient with those findings should be considered as having hypertensive retinopathy and choroidopathy and treated as soon as possible because of the poor prognostic.

Conclusion: Hypertensive choroidopathy is a rare finding associated with acute increases in blood pressure. When the choroid is associated, the hypertensive event is often more acute and associated with increased morbidity. It is necessary to obtain fundus exam in any patient with elevated blood pressure and concomitant vision complaints. Therefore, screening hypertensive patients involves close collaboration between internist and ophthalmologist.

Keywords: Hypertensive choroidopathy, hypertensive retinopathy, exudative retinal detachment, elschnig's spot, siegrist streak, chronic kidney disease

Cite This Article: KARTIKASARI, indha dwi; DEWI, Nadia Artha. Bilateral Exudative Retinal Detachment Due to Hypertensive Retinopathy and Choroidopathy In Young Patient with Chronic Kidney Disease. International Journal of Retina, [S.I.], v. 1, n. 1, july 2018. ISSN 2614-8536. Available at: <https://www.ijretina.com/index.php/ijretina/article/view/20>

*Correspondence to: Indha Dwi Kartikasari, Department of Ophthalmology, Universitas Brawijaya, indhadk@gmail.com

\section{INTRODUCTION}

Severe systemic hypertension in chronic kidney disease can cause significant damage to the eye. Although hypertensive retinopathy is a well-known complication, hypertensive neuropathy and choroidopathy are much less common. Elevation of systemic blood pressure as in renovascular disease causes both focal and generalized retinal arteriolar constriction, presumably mediated by autoregulation. A prolonged duration of particularly high blood pressure can be associated with a breakdown of the inner blood-retinal barrier, with extravasation of plasma and red blood cells. Retinal hemorrhages, cottonwool spots, intraretinal lipid, and, in severe cases, the development of a macular star configuration of intraretinal lipid can be seen ${ }^{1-3}$. When the choroidal vessels are severely affected by elevated blood pressure, as in acute hypertension, fibrinoid necrosis of choroidal arterioles can cause occlusion of areas of choriocapillaris, with a subsequent breakdown of the outer blood-retinal barrier. Although the retinal vascular changes and optic neuropathy are well known, hypertensive choroidopathy usually does not receive as much attention. Hypertensive choroidopathy has been reported in toxemia of pregnancy, renal disease, pheochromocytoma, and malignant 
Hypertension retinopathy occur almost half in CKD population, while hypertension choroidopathy only on $0,1 \%^{5}$. We report a case of hypertensive retinopathy and hypertensive choroidopathy with bilateral exudative retinal detachments in young patient with chronic kidney disease.

\section{CASE ILLUSTRATION}

A 26-year-old man presented with a painless loss of vision in his both eyes. He had a history of chronic kidney disease (CKD) gr-V on hemodyalisis since 2 months ago. Ophthalmic examination revealed visual acuity of $0.5 / 60$ OU. Pupils reacted normally without an afferent pupillary defect. He had normal extraocular motility, and ocular alignment in both eyes. Slit lamp examination revealed subconjunctival haemorrhage in both eyes. Retinal examination revealed retinal arteriolar narrowing, vascular tortuosity, arteriovenous nicking, optic disc swelling, retinal hemorrhage, intraretinal exudation and macular star formation, exudative retinal detachment, Elsching spot, and Siegrist streak. The blood pressure was 200/140 $\mathrm{mmHg}$. Hemoglobin $8,0 \mathrm{~g} / \mathrm{dL}, \mathrm{RBC} 2,96 \times 10^{6} / \mu \mathrm{L}, \mathrm{WBC}$ 13.130/ $\mu \mathrm{L}$, hematocrit $24,10 \%$, PLT $114.000 / \mu \mathrm{L}$, RBS 150 $\mathrm{mg} / \mathrm{dL}$, ureum 249,70 mg/dL, creatinin $20,61 \mathrm{mg} / \mathrm{dL}$. Urinalysis $\mathrm{pH} 8,5$; density 1,015 ; glucose $1+$; protein $3+$; keton, bilirubin, urobilinogen, nitrit negative; WBC $2+$, blood 3+; epithel 1,1 LPK, cylinder negative; RBC 41,9 LPB; WBC 46,5 LPB, crystal negative, bacteria $2750,8 \times 10^{3} / \mathrm{mL}$, shown in the laboratory result. The patient was diagnosed with bilateral hypertensive retinopathy and choroidopathy with bullous exudative retinal detachment and treated with antihypertensive treatment glyceryl trinitrate (GTN) drip 20-200 $\mu / \mathrm{mnt}$ and furosemide drip $10 \mathrm{mg} /$ hour, amlodipine $1 \times 10 \mathrm{mg}$ tab, valsartan $1 \times 80 \mathrm{mg}$ tab, lansoprazole $1 \times 30 \mathrm{mg}$ intravena (iv), and metoclopramide $3 \times 10 \mathrm{mg}$ iv.

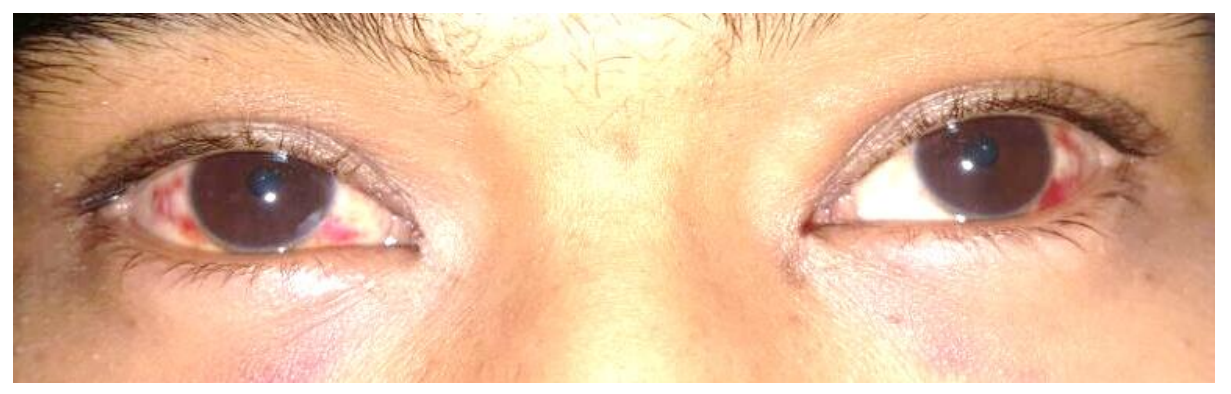

Figure 1. Subkonjungtival Haemorrhage on Both Eyes

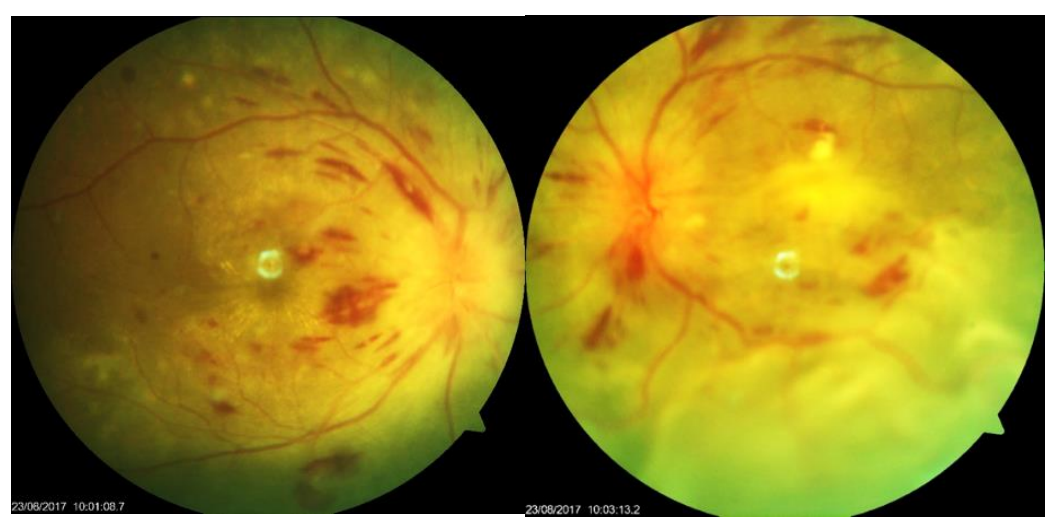

Figure 2. Fundus Photograhy Show Bilateral Disc Edema, Retinal Bleeding, Exudative Retinal Detachment, Elschnig Spot, Siegrist Streak, and Macular Star (Courtesy By Vitreo-Retina Division)

Patient came at outpatient clinic after 2 weeks with improvement in visual acuity to $1 / 60$ with better retinal findings, but decreasing in general condition. According to systemic condition, 2 weeks later the patient passed away.

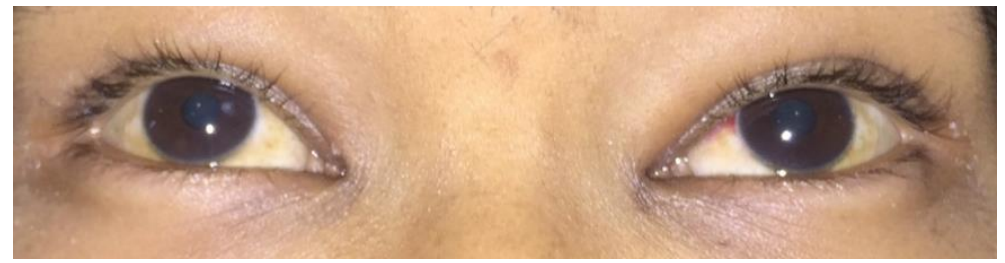

Figure 3. There Were Reducing of Subconjunctival Haemorrhage on Both Eyes After 3 Weeks 


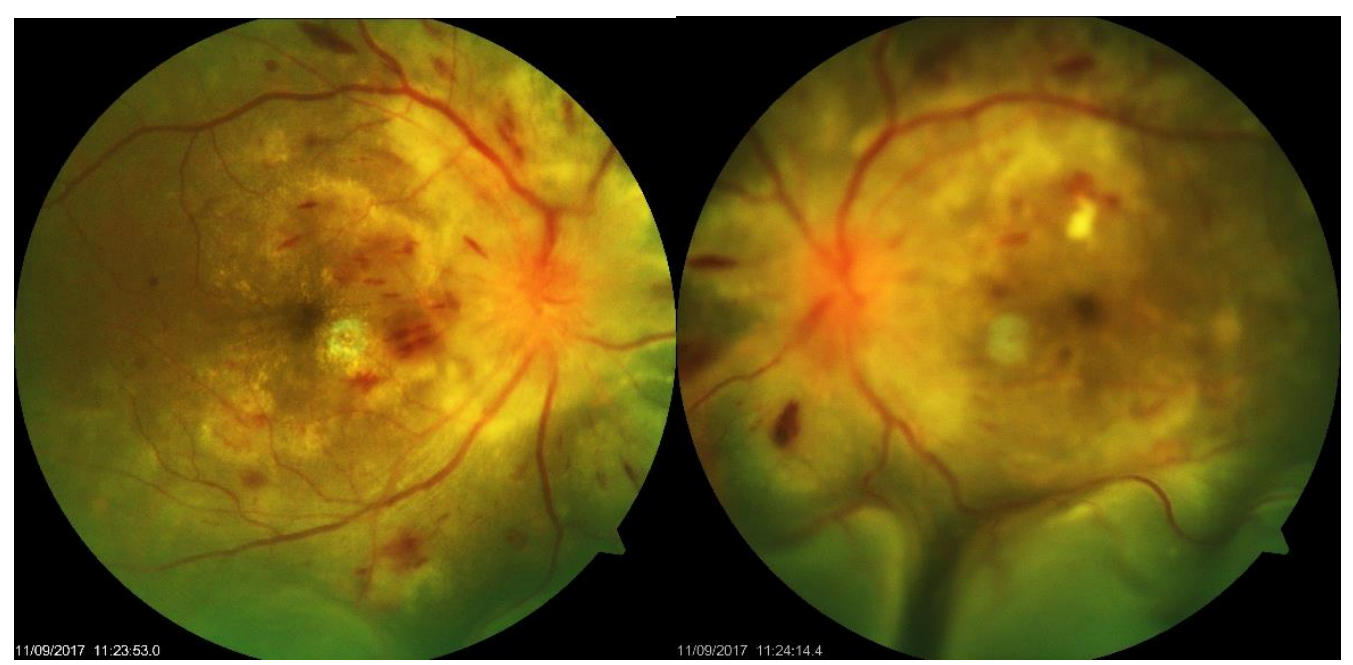

Figure 4. Fundus Photography After 3 Weeks Show Reducing Of Bilateral Disc Edema, Retinal Bleeding, And Exudative Retinal Detachment(Courtesy by Vitreo-retina Division)
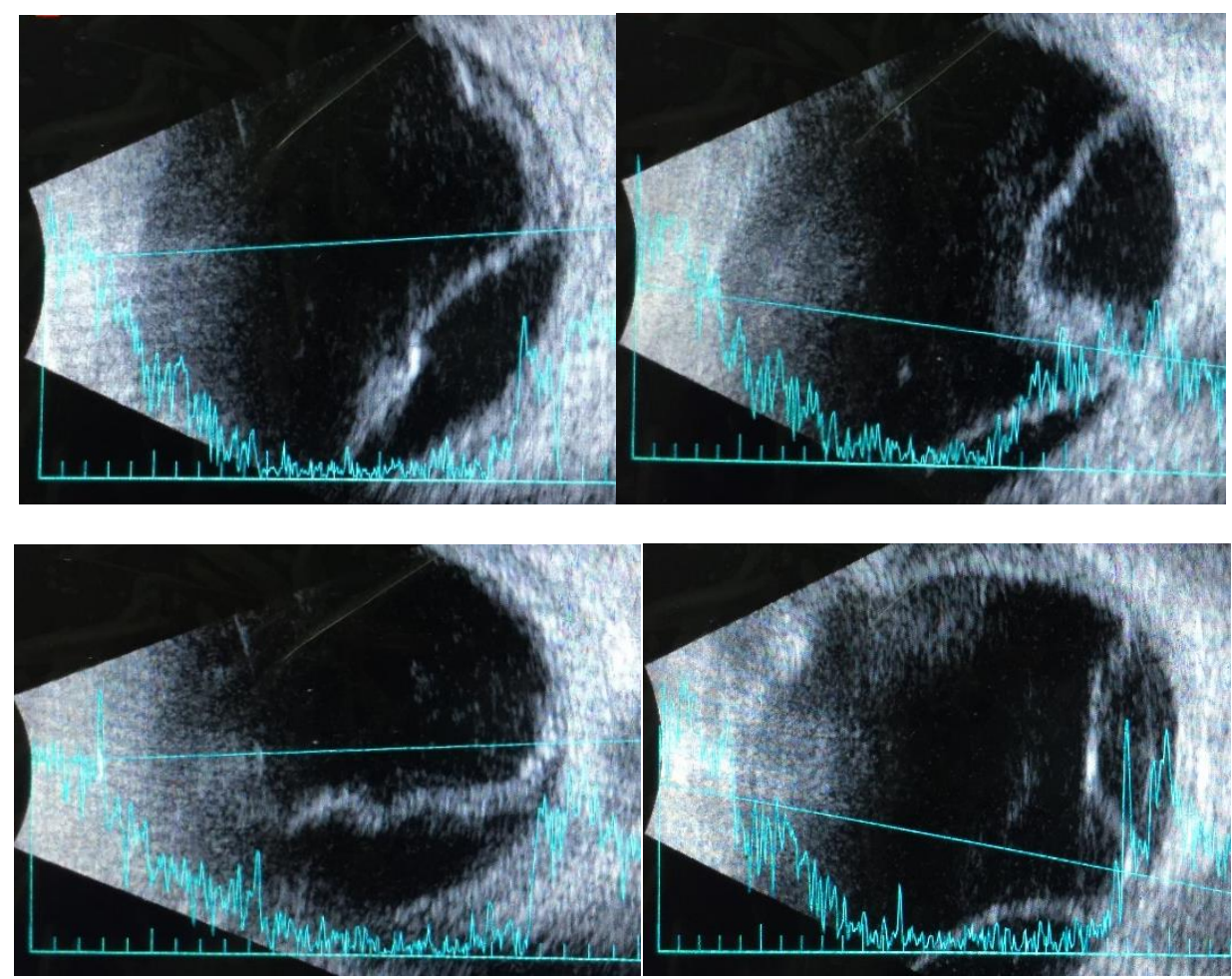

Figure 5. Ocular USG Right Eye (Above) Left Eye (Below) When Sitting Postion (Left Photo) and Supine Position (Right Photo) Showed Shifting Fluid As Gravitation. (Courtesy By Vitreo-Retina Division)

\section{DISCUSSION}

Severe systemic hypertension is associated with significant damage of end organs, such as eyes, heart, central nervous system, and kidneys. Choroidal lesions are less commonly recognized than retinal and optic nerve lesions ${ }^{6}$. Subconjunctival haemorrhage associated with valsava maneuver and drastically increased blood pressure ${ }^{7}$ Accelerated hypertension and/or ophthalmic/ciliary artery occlusion may result in choroidal ischemia. With complete ophthalmic artery occlusion, both the retinal and choroidal circulations are compromised. Compromise of ciliary arteries may occur without retinal artery involvement, leading to choroidal ischemia, which causes hypertensive choroidopathy. Some of these are attributed to a breakdown of the inner blood-retinal barrier with retinal endothelial cell decompensation. Furthermore, there are large fenestrations in the walls of the choriocapillaris, so the choroidal vascular bed has no blood ocular barrier. This causes leakage of plasma into the choroidal fluid. Angiotensin II and other vasoconstrictors in the choroidal fluid result in vasoconstriction and ischemia of the choroidal vasculature. This cascade is followed by RPE ischemia and the subsequent breakdown of the blood retinal barrier in the RPE and result as Elcshnig spot and 
Siegrist streak. The RPE becomes necrotic, this may result in exudative retinal detachment ${ }^{8}$.

The narrowed arterioles, vascular tortuosity, and arteriolovenous nicking in the retinal vessels were also observed. These findings are relatively common in longstanding hypertension. It seems that the patient had a history of longstanding chronic hypertension, and a sudden BP elevation triggered it off and formed this condition. After antihypertension treatment, the exudative retinal detachment and others condition remain still. In most cases with hypertensive choroidopathy, visual acuity returns to normal by controlling their BP. However, there is a case reporting that the visual acuity was not recovered in spite of being controlled the $\mathrm{BP}^{9}$. A patient with these findings should be considered as hypertensive retinopathy and choroidopathy and treated with antihypertension therapy as soon as possible. In exudative retinal detachment, when the fluid is not resolving and retinal detachment is chronic and bullous, permanent damage to retinal pigment epithelium as well as outer retinal structures occurs and persistence of subretinal fibrin may lead to subretinal fibrotic scar formation. To avoid these complications, surgical intervention either external drainage or pars plana vitrectomy is planned after failure of conventional treatment. However, surgical treatment is never the first line of management in these conditions $^{10}$. This disease has poor prognosis caused by continuous process during the damage of blood vessel, even if the BP is in a good state, the complication still occurs in $50 \%$ patient ${ }^{9}$. Hypertensive choroidopathy is a less-common complication of systemic hypertension but can be the harbinger of a potentially life-threatening hypertensive emergency with end-organ damage. Choroidal involvement in the setting of hypertensive emergency is usually the sign of an acute, dramatic increase in systemic blood pressure in a young person and associated with poor visual and systemic prognosis.

\section{CONCLUSION}

Hypertensive choroidopathy is a rare finding associated with acute increases in blood pressure. When the choroid is involved, the hypertensive event is often more acute and associated with increased morbidity. It is necessary to obtain a fundus exam in any patient with elevated blood pressure and concomitant vision complaints. Therefore, screening hypertensive patients involves close collaboration between internist and ophthalmologist. Antihypertensive is the first line treatment, but if the exudative retinal detachment not resolve and retinal detachment is chronic and bullous, surgical intervention to drain the fluid can be planned.

\section{REFERENCES}

1. A. G. Lee and H. A. Beaver, "Acute bilateral optic disk edema with a macular star figure in a 12year-old girl," Survey of Ophthalmology, vol. 47, no. 1, pp. 42-49, 2002.

2. A. Grosso, F. Veglio, M. Porta, F. M. Grignolo, and T. Y. Wong, "Hypertensive retinopathy revisited: some answers, more questions," British Journal of Ophthalmology, vol. 89, no. 12, pp. 1646-1654, 2005.

3. T. Y. Wong and P. Mitchell, "Hypertensive retinopathy," The New England Journal of Medicine, vol. 351, no. 22, pp. 2310-2317, 2004.

4. M. O. M. Tso and L. M. Jampol, "Pathophysiology of hypertensive retinopathy," Ophthalmology, vol. 89, no. 10, pp. 1132-1145, 1982.

5. Bajracharya L, Shah D, Rait K, Koirala S. Ocular Evaluation In Patients With Chronic Renal FailureA Hospital Based Study. Nepal Med Coll J, 2008;10(4)209-214

6. B. P. Luo and G. C. Brown, "Update on the ocular manifestations of systemic arterial hypertension," Current Opinion in Ophthalmology, vol. 15, no. 3, pp. 203-210, 2004.

7. Shoji Kishi, Mark O. M. Tso dan Hayreh, Sohan Singh. Fundus Lesions in Malignant Hypertension: A Pathologic Study of Experimental Hypertensive Optic Neuropathy. Arch Ophthalmol. 1985;103(8):1198-1206

8. Hayreh, Sohan Singh. Ocular Vascular Occlusive Disorders. New York: Springer, 2015

9. M. Ugarte, S. Horgan, S. Rassam, T. Leong, and C. H. Kon, "Hypertensive choroidopathy: recognizing clinically significant end-organ damage," Acta Ophthalmologica, vol. 86, no. 2, pp. 227-228, 2008.

10. Ganesan S. and Rishi E. Surgical implications in exudative retinal detachment, Sci J Med \& Vis Res Foun 2017;XXXV:29-36.

\section{@ $\odot \Theta \Theta$}

This work licensed under Creative Commons Attribution 